\title{
Nanoscale Correlative Imaging of Low-Z elements by in-situ Secondary Ion Mass Spectrometry in a Transmission Electron Microscope
}

\author{
Santhana Eswara ${ }^{1 *}$, Jelena Lovric ${ }^{1}$ and Tom Wirtz ${ }^{1}$ \\ 1. Advanced Instrumentation for Ion Nano-Analytics (AINA), MRT Department, Luxembourg Institute \\ of Science and Technology, 41, rue du Brill, L-4422 Belvaux, Luxembourg. \\ * Corresponding author: santhana.eswara@list.lu
}

Transmission Electron Microscopy (TEM) is a well-established technique for high-resolution imaging down to atomic scale [1]. However, analyses of low-Z elements $(Z<6)$ are notoriously difficult or even impossible using the typical analytical tools associated with TEM such as Energy Dispersive X-Ray Spectroscopy or Electron Energy-Loss Spectroscopy. In comparison, Secondary Ion Mass Spectrometry (SIMS) imaging is a well-known high-sensitivity technique for the analysis of all elements of the periodic table including their isotopes [2]. However, the lateral resolution of SIMS imaging is fundamentally limited by the ion-solid interaction volume to $\sim 10 \mathrm{~nm}$, which is 2 to 3 orders of magnitude poorer in comparison to TEM imaging. In order to combine the benefits of TEM and SIMS in a single instrument, we developed an in-situ TEM-SIMS technique [3].

The octagon of a FEI Tecnai G2 F20 TEM was adapted to integrate a Focused Ion Beam (FIB) column and an in-house designed and built compact mass spectrometer (Fig. 1). The FIB is a FEI Magnum column with monoisotopic ${ }^{69} \mathrm{Ga}^{+}$source. A special TEM sample holder with a tip that can be biased up to $\pm 4.5 \mathrm{kV}$ was developed to enhance the collection efficiency of the secondary ions. With this set up, TEM and SIMS images can be obtained within the same instrument, thus decreasing the analysis time. Furthermore, artefacts associated with sample transfer between different stand-alone instruments, such as sample modifications and non-systematic image distortions, etc. are avoided. The correlative TEMSIMS datasets (e.g. Fig. 2) thus obtained can then be treated by image fusion methods to derive new insights not achieved by the individual techniques [4].

In this presentation, our recent results related to the analysis of low- $Z$ elements in materials science applications will be discussed. A special focus will be on the imaging and analysis of hydrogen $(Z=1)$ (including deuterium), and boron $(Z=5)$ in silicon solar cells. Additionally, we will also discuss the imaging of lithium $(Z=3)$ and other elements in cathode materials of cycled and uncycled lithium-ion batteries [5].

References:

[1] DB Williams and CB Carter, The Transmission Electron Microscope (Springer US, Boston, MA, 2009).

[2] A Benninghoven, FG Rüdenauer, and HW Werner, Secondary Ion Mass Spectrometry : Basic Concepts, Instrumental Aspects, Applications, and Trends (J. Wiley, New York, 1987).

[3] L Yedra et al., Sci. Rep. 6 (2016), p. 28705.

[4] F Vollnhals et al., Anal. Chem. 89 (2017), p. 10702.

[5] The authors acknowledge funding from the LowZ-PIES (C13/MS/5951975) and the NACHOS (INTER/SNF/16/11536628) projects funded by the Luxembourg National Research Fund (FNR) by the grant. 

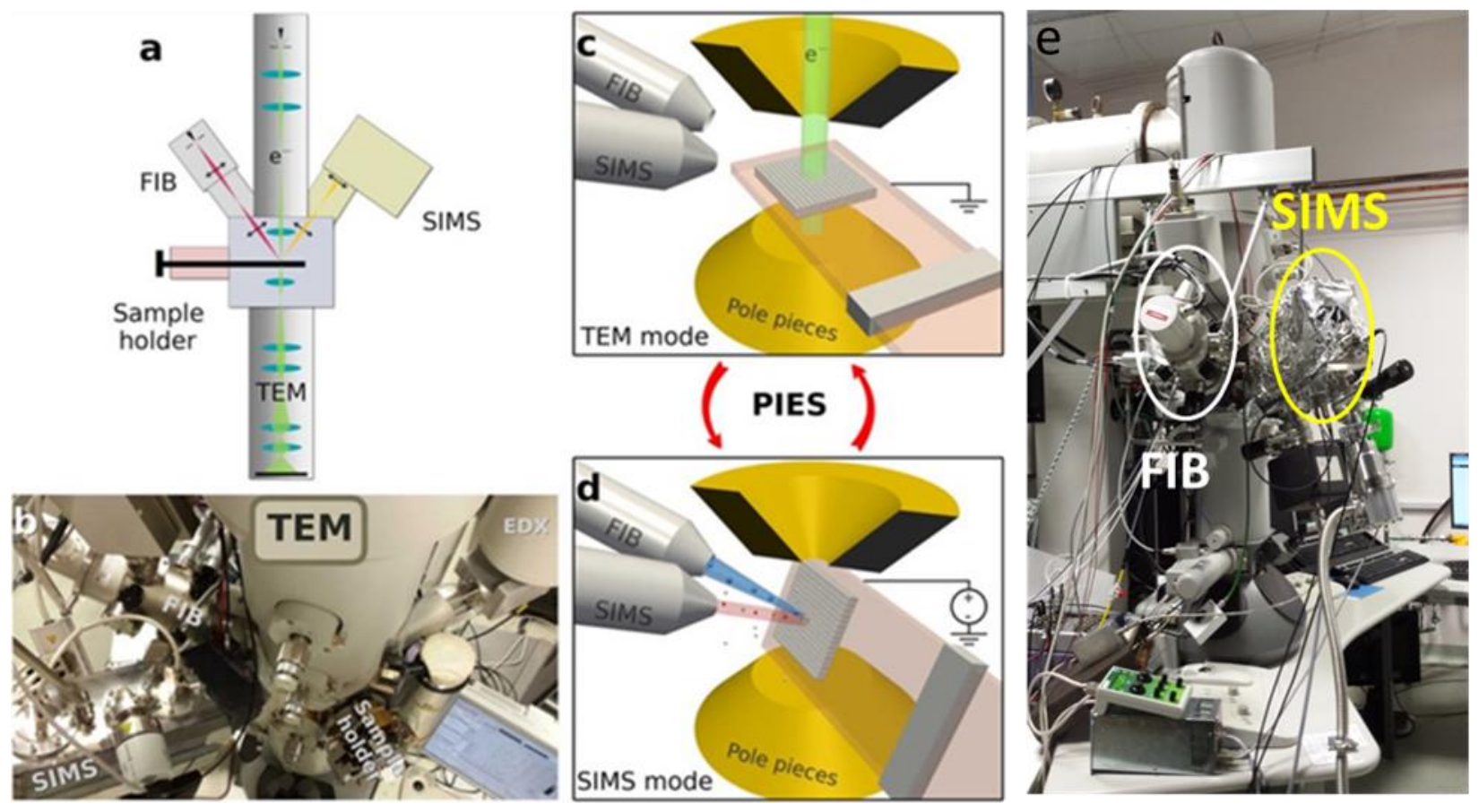

Figure 1. The layout (a), photos (b, e) and schematics (c, d) of the in-situ TEM-SIMS instrument (Adapted from [3]).
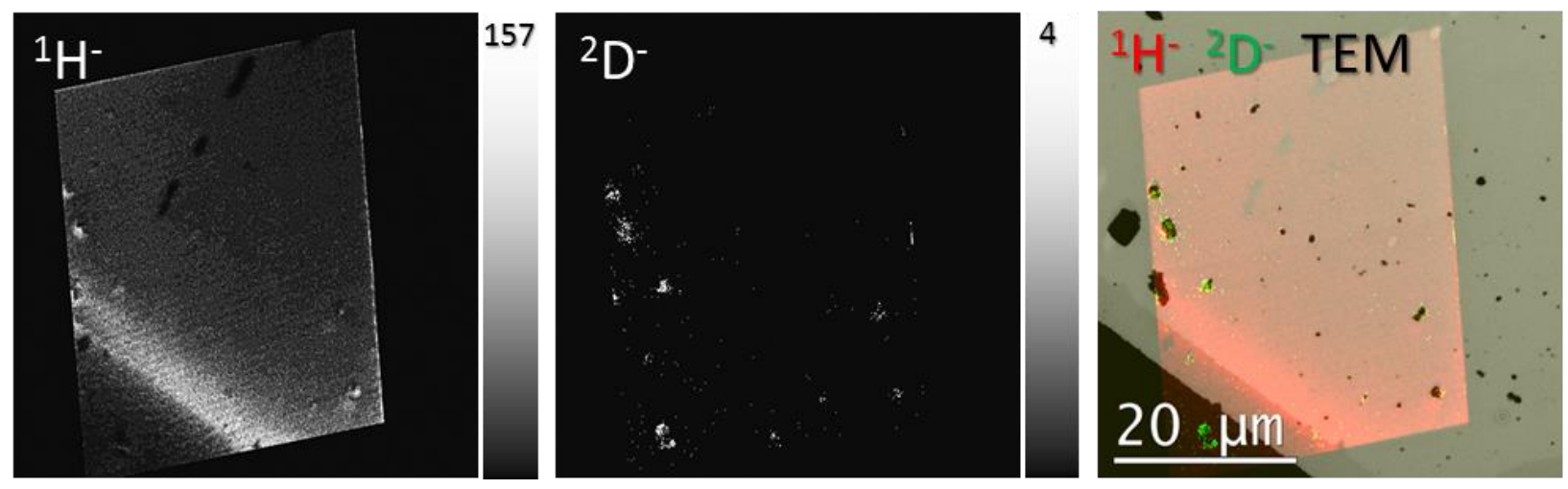

Figure 2. In-situ SIMS images $\left(\mathrm{H}^{-}\right.$and $\left.\mathrm{D}^{-}\right)$superposed on a corresponding TEM image from a sample containing a physical mixture $\mathrm{KD}_{2} \mathrm{PO}_{4}$ and $\mathrm{KH}_{2} \mathrm{PO}_{4}$ powders. Because of the non-UHV conditions in the TEM, the $\mathrm{H}$ map has a higher background level than the D map. 